YeastOne-Sensititre, in accordo con il documento M27-A dell'NCCLS.

Risultati. Sono stati raccolti 58 ceppi da differenti campioni: emocolture, CVC, tamponi uretrali, aspirati, escreati, urine e feci. C. albicans rappresenta la specie maggiormente isolata con una percentuale pari a $81 \%$, a cui fan seguito C. tropicalis $(10,3 \%)$, C. glabrata $(5,2 \%)$ e C. parapsilosis $(3,5 \%)$.

Amfotericina B e 5-fluorocitosina hanno dimostrato efficacia verso i lieviti testati, con uguali valori di $\mathrm{MIC}_{50}(0,06 \mu \mathrm{g} / \mathrm{ml})$ e $\mathrm{MIC}_{90}$ pari rispettivamente a $0,125 \mu \mathrm{g} / \mathrm{ml}$ e $0,25 \mu \mathrm{g} / \mathrm{ml}$. Voriconazolo e chetoconazolo si sono dimostrati i composti maggiormente attivi $\left(\mathrm{MIC}_{50} \leq 0,008 \mu \mathrm{g} / \mathrm{ml} ; \mathrm{MIC}_{90}\right.$ $0,125 \mu \mathrm{g} / \mathrm{ml}$ ); una sensibilità dose-dipendente al fluconazolo è emersa in due isolati di C. albicans e C.glabrata e uno di C. parapsilosis. Due ceppi di C. tropicalis hanno mostrato resistenza verso questa molecola.

Conclusioni. C.albicans rimane il patogeno maggiormente isolato dalle infezioni profonde della nostra area geografica, nonostante una rilevante presenza di specie non-albicans. Come osservato in altri studi, la sensibilità al fluconazolo è risultata specie-correlata; perciò è opportuno sottolineare l'importanza dell'identificazione a livello di specie dei lieviti di isolamento clinico.

\title{
PREVALENZA E SENSIBILITÀ IN VITRO AGLI ANTIFUNGINI DI ISOLATI DI CANDIDA SPP. DA MICOSI PROFONDE
}

\author{
Asticcioli S. ${ }^{a}$, Sacco L. ${ }^{b}$, Cardillo A. ${ }^{b}$, Pagani L. ${ }^{a}$ \\ ${ }^{a}$ Dipartimento S.M.E.C. Sezione di Microbiologia, \\ Università degli Studi di Pavia, via Brambilla 74, 27100 Pavia. \\ "Servizio Analisi Microbiologiche I.R.C.C.S. "S.Matteo", \\ Viale Golgi 19, 27100 Pavia.
}

Introduzione. Negli ultimi anni si è assistito ad un incremento dell'incidenza delle infezioni sistemiche ad eziologia fungina ed i funghi unicellulari, specialmente i lieviti appartenenti al genere Candida, rappresentano i più comuni agenti di tali micosi. Lo spettro d'infezioni causate da questi lieviti ha subito un cambiamento dovuto ad un aumento percentuale delle specie non-albicans ed alla diminuizione d'isolamento di C. albicans, sebbene rimanga il lievito maggiormente responsabile di micosi.

Scopo dello studio è stato determinare frequenza, distribuzione di specie e profilo di farmaco-sensibilità di ceppi di Candida isolati da infezioni profonde durante il periodo 20032005 da differenti reparti dell'IRCCS S. Matteo di Pavia.

Metodi. I lieviti sono stati identificati mediante caratterizzazione del profilo biochimico-metabolico (VITEK system e API 20CAUX - BioMérieux). I saggi di sensibilità sono stati condotti avvalendosi del metodo di microdiluizione in brodo 